\title{
EXAMPLES OF COMPACT $K$-CONTACT MANIFOLDS WITH NO SASAKIAN METRIC
}

\author{
BENIAMINO CAPPELLETTI-MONTANO, ANTONIO DE NICOLA, \\ JUAN CARLOS MARRERO, AND IVAN YUDIN
}

\begin{abstract}
Using the Hard Lefschetz Theorem for Sasakian manifolds, we find two examples of compact $K$-contact nilmanifolds with no compatible Sasakian metric in dimensions five and seven, respectively.
\end{abstract}

\section{INTRODUCTION}

Construction of examples of compact symplectic manifolds with no Kähler structures is a topic which attracted wide interest in recent years (see e.g. [17] and reference therein). Many techniques have been used to identify such examples. The first technique used to prove that some examples were non-Kähler consists in showing that some odd Betti number of the manifold is not even and thus the manifold cannot be Kähler (see e.g. [16]). In other cases (e.g. [2]), when the all odd Betti numbers are even, one possibility to prove that a certain symplectic manifold cannot admit a Kähler structure is to show that it does not satisfy the Hard Lefschetz theorem.

In odd dimensions, a Hard Lefschetz theorem for compact co-Kähler manifolds was proven in 6 . However, until recently only the Betti number technique was available to show that a compact $K$-contact manifold does not admit any Sasakian structure. An example of a compact $K$-contact manifold with no Sasakian structure was found by Boyer and Galicki in [4, Example 7.4.16]. They constructed a non-trivial $\mathbb{T}^{3}$ bundle over $\mathbb{T}^{2}$ and proved that it is non-Sasakian since the first Betti number is odd. Recently a Hard Lefschetz theorem for compact Sasakian manifolds was proven ([5]).

In this paper we give two examples of compact co-orientable $K$-contact manifolds with no Sasakian structure compatible with the contact one. The examples, in dimensions five and seven respectively, are intended as first applications of our Hard Lefschetz theorem. We take advantage of the fact that lately Kutsak has classified the invariant contact structures on nilmanifolds up to dimension seven in [11. Thus, by using this classification one can construct examples of compact contact nilmanifolds in any odd dimension 3,5, 7. Actually, since the only 3-dimensional nilpotent Lie algebra admitting an invariant contact structure is the Heisenberg algebra, the non-trivial dimensions are 5 and 7 . Among these examples we find one in dimension 5 with $b_{1}=2$ and one in dimension 7 with $b_{1}=4$ and $b_{3}=8$. Thus, the Betti numbers data do not give us any obstruction for the manifolds to admit a Sasakian metric. However, we prove that, while both nilmanifolds are endowed with a left-invariant $K$-contact structure, they cannot admit any Sasakian metric compatible with the underlying contact structure since they do not satisfy the Hard Lefschetz Theorem.

Concerning the question of finding non-trivial examples of $K$-contact manifolds which cannot carry any Sasakian metric, we mention the very recent paper [9], where compact and simply connected examples in dimensions $\geq 11$ are found by using some 
geometric techniques based on the notion of contact fatness developed by Lerman in [12] and [13]. However, we notice that in [9] the fact that the manifold is nonSasakian was established by proving that the third Betti number is odd. Finally, when the present paper was in the final stages of preparation, a new result appeared in 15. where examples of simply connected $K$-contact manifolds without any Sasakian structures in dimensions $\geq 9$ are found by using the Hard Lefschetz Theorem.

\section{Preliminaries}

In this section we recall some basic definitions and properties in contact Riemannian geometry. For further details we refer the reader to the monographs [3] or [4].

Let $M$ be a smooth manifold of dimension $2 n+1$. A 1 -form $\eta$ on $M$ is called a contact form if $\eta \wedge \mathrm{d} \eta^{n}$ is a volume form. Then the pair $(M, \eta)$ is called a (strict) contact manifold. In any contact manifold one proves the existence of a unique vector field $\xi$, called the Reeb vector field, satisfying the properties

$$
i_{\xi} \eta=1, \quad i_{\xi} \mathrm{d} \eta=0 .
$$

Given a contact manifold $(M, \eta)$ there always exists a Riemannian metric $g$ and a tensor field $\phi$ of type $(1,1)$ such that the following conditions hold

$$
\begin{gathered}
\eta=g(\cdot, \xi), \\
\mathrm{d} \eta=2 g(\cdot, \phi \cdot), \\
\phi^{2}=-I+\eta \otimes \xi,
\end{gathered}
$$

where $I: T M \rightarrow T M$ denotes the identity mapping. From (2.1)-(2.3) it follows that $\phi \xi=0, \eta \circ \phi=0$ and

$$
g(\phi X, \phi Y)=g(X, Y)-\eta(X) \eta(Y)
$$

for any $X, Y \in \Gamma(T M)$. Moreover, (2.2) implies that the bilinear form $\Phi:=g(\cdot, \phi \cdot)$ is in fact a 2-form, which is sometimes called Sasaki form. The manifold $M$ together with the above geometric structure $(\phi, \xi, \eta, g)$ is called a contact metric manifold.

A Sasakian manifold is a contact metric manifold for which the following normality condition is satisfied

$$
[\phi, \phi]_{F N}+2 \mathrm{~d} \eta \otimes \xi=0,
$$

where $[-,-]_{F N}$ is the Frölicher-Nijenhuis bracket (see e.g. [10]).

An equivalent way to express the Sasakian condition is to say that the Riemannian cone of $(M, \eta, g)$ is a Kähler manifold.

In any Sasakian manifold the Reeb vector field is Killing. This last property is equivalent to the condition $\mathcal{L}_{\xi} \phi=0$. More generally, a contact metric manifold whose Reeb vector field is Killing is called $K$-contact. Thus any Sasakian manifold is $K$-contact and it is known that the converse holds in dimension 3.

A well-known obstruction to the existence of a Sasakian structure on a contact manifold $(M, \eta)$ is given by the following theorem, due to Fujitani.

Theorem $2.1([8])$. Let $(M, \eta)$ be a compact contact manifold of dimension $2 n+1$. If $M$ admits a compatible Sasakian structure, then for any odd integer $p \leq n+1$ the Betti numbers $b_{p}$ are even.

In [5] an obstruction stronger than the one expressed in Theorem 2.1 was found, by proving an odd dimensional counterpart of the celebrated Hard Lefschetz Theorem. 
Theorem $2.2([\underline{5})$. Let $(M, \phi, \xi, \eta, g)$ be a compact Sasakian manifold of dimension $2 n+1$. Then for each integer $0 \leq p \leq n$ the maps

$$
\begin{aligned}
\operatorname{Lef}_{n-p}: H^{n-p}(M) & \rightarrow H^{n+p+1}(M) \\
{[\beta] } & \mapsto\left[\eta \wedge(\mathrm{d} \eta)^{p} \wedge \Pi_{\Delta} \beta\right],
\end{aligned}
$$

are isomorphisms, $\Pi_{\Delta} \beta$ denoting the orthogonal projection of $\beta$ on the space of harmonic forms.

Notice that, contrary to the even dimensional case, it is not true that the wedge multiplication by $\eta$ or by $\eta \wedge \mathrm{d} \eta$ maps harmonic forms into harmonic forms, so that one is forced to use the metric in order to define the Lefschetz maps (2.4). Thus, a priori, one could expect that different Sasakian metrics, all compatible with the same underlying contact form, could lead to different Lefschetz isomorphisms. However, in [5. Theorem 4.5] it is proved that the Lefschetz isomorphisms are independent of the metric. The proof of [5, Theorem 4.5] suggests to introduce the notion of contact Lefschetz manifold. Namely, according to [5, let us define the Lefschetz relation between cohomology groups $H^{p}(M)$ and $H^{2 n+1-p}(M)$ of a contact manifold $(M, \eta)$ to be

$$
\mathcal{R}_{\text {Lef }_{p}}=\left\{\left([\beta],\left[\epsilon_{\eta} L^{n-p} \beta\right]\right) \mid \beta \in \Omega^{p}(M), \mathrm{d} \beta=0, i_{\xi} \beta=0, L^{n-p+1} \beta=0\right\} .
$$

Here, given $\alpha \in \Omega^{k}(M)$, the operator $\epsilon_{\alpha}$ is defined by

$$
\epsilon_{\alpha} \beta=\alpha \wedge \beta
$$

$L=\epsilon_{\frac{1}{2} \mathrm{~d} \eta}$ and the conditions $\mathrm{d} \beta=0, i_{\xi} \beta=0$, and $L^{n-p+1} \beta=0$ imply that the $((2 n+1)-p)$-form $\epsilon_{\eta} L^{n-p} \beta$ is closed and $\left[\epsilon_{\eta} L^{n-p} \beta\right]=\operatorname{Lef}_{p}[\beta]$ (see Theorem 4.5 in [5]). Therefore if $(M, \eta)$ admits a compatible Sasakian metric, due to Theorem 2.2 it follows that $\mathcal{R}_{L e f_{p}}$ is the graph of the isomorphism $\operatorname{Lef}_{p}$. More generally, a compact contact manifold $(M, \eta)$ is said to be Lefschetz contact if it satisfies the hard Lefschetz property, that is, for every $p \leq n$ the relation $\mathcal{R}_{\text {Lef }_{p}}$ is the graph of an isomorphism between $H^{p}(M)$ and $H^{2 n+1-p}(M)$.

We point out that, according to [5. Theorem 5.2], every Lefschetz contact manifold satisfies the restrictions on the Betti numbers for compact Sasakian manifolds stated in Theorem 2.1.

\section{EXAmples of COMPaCT $K$-CONTACT MANifoldS With NO COMPATIBle SASAKIAN METRIC}

Hereinafter, by using the aforementioned Hard Lefschetz Theorem for Sasakian manifolds, we shall present two examples of compact contact manifolds (actually, $K$-contact manifolds) of dimension 5 and 7 , respectively, which do not admit any compatible Sasakian metric. As we shall see, one cannot deduce the assertion from the well-known restrictions concerning the Betti numbers of the manifold.

Example 3.1. Let us consider the 5-dimensional nilpotent Lie algebra $\mathfrak{g}$ with non-zero Lie brackets

$$
\left[X_{1}, X_{2}\right]=X_{3}, \quad\left[X_{1}, X_{3}\right]=X_{4}, \quad\left[X_{1}, X_{4}\right]=X_{5}, \quad\left[X_{2}, X_{3}\right]=X_{5} .
$$


Let us denote by $\alpha_{i}$ the dual 1 -form of the vector $X_{i}$, for any $i \in\{1, \ldots, 5\}$. Then by (3.1) it follows that

$$
\begin{aligned}
& \mathrm{d} \alpha_{1}=0, \quad \mathrm{~d} \alpha_{2}=0, \quad \mathrm{~d} \alpha_{3}=-\alpha_{1} \wedge \alpha_{2}, \quad \mathrm{~d} \alpha_{4}=-\alpha_{1} \wedge \alpha_{3}, \\
& \mathrm{~d} \alpha_{5}=\alpha_{1} \wedge \alpha_{4}+\alpha_{2} \wedge \alpha_{3} .
\end{aligned}
$$

Here, $\mathrm{d}: \wedge^{k} \mathfrak{g}^{*} \rightarrow \wedge^{k+1} \mathfrak{g}^{*}$ is the Chevalley-Eilenberg differential in the Lie algebra $\mathfrak{g}$. We have $\alpha_{5} \wedge\left(\mathrm{d} \alpha_{5}\right)^{2}=2 \alpha_{1} \wedge \alpha_{2} \wedge \alpha_{3} \wedge \alpha_{4} \wedge \alpha_{5}$, hence if $\eta$ is the left-invariant 1-form induced by $\alpha_{5}$ on the simply connected Lie group $G$ whose Lie algebra is $\mathfrak{g}$, then $\eta$ is a contact 1-form on $G$. The corresponding Reeb vector field is the left-invariant vector field $\xi$ on $G$ induced by $X_{5}$.

Now, let us consider a co-compact discrete subgroup $\Gamma$ of $G$. Such a subgroup exists by the Malcev's criterion, since in our case the structure constants are integers. Then the quotient $M=G / \Gamma$ is a compact nilmanifold.

Remark 3.2. The Lie group $G$ is isomorphic to $\mathbb{R}^{5}$ endowed with the multiplication defined by

$$
\begin{aligned}
\left(x^{1}, x^{2}, x^{3}, x^{4}, x^{5}\right) \cdot & \left(y^{1}, y^{2}, y^{3}, y^{4}, y^{5}\right)=\left(x^{1}+y^{1}, x^{2}+y^{2}, x^{3}+y^{3}+x^{1} y^{2}, x^{4}+y^{4}\right. \\
+ & x^{1} y^{3}+\frac{\left(x^{1}\right)^{2}}{2} y^{2}, x^{5}+y^{5}-x^{1} y^{4}-\left(\frac{\left(x^{1}\right)^{2}}{2}+x^{2}\right) y^{3} \\
& \left.-\frac{x^{1}}{2}\left(y^{2}\right)^{2}-\left(\frac{\left(x^{1}\right)^{3}}{6}+x^{1} x^{2}\right) y^{2}\right)
\end{aligned}
$$

for $\left(x^{1}, x^{2}, x^{3}, x^{4}, x^{5}\right),\left(y^{1}, y^{2}, y^{3}, y^{4}, y^{5}\right) \in \mathbb{R}^{5}$. A basis of the space of the left-invariant 1 -forms is given by

$$
\begin{aligned}
& \alpha_{1}=\mathrm{d} x^{1}, \quad \alpha_{2}=\mathrm{d} x^{2}, \quad \alpha_{3}=\mathrm{d} x^{3}-x^{1} \mathrm{~d} x^{2}, \quad \alpha_{4}=\mathrm{d} x^{4}-x^{1} \mathrm{~d} x^{3}+\frac{\left(x^{1}\right)^{2}}{2} \mathrm{~d} x^{2}, \\
& \alpha_{5}=\mathrm{d} x^{5}+x^{1} \mathrm{~d} x^{4}-\frac{\left(x^{1}\right)^{2}}{2} \mathrm{~d} x^{3}+\frac{\left(x^{1}\right)^{3}}{6} \mathrm{~d} x^{2}+x^{2} \mathrm{~d} x^{3} .
\end{aligned}
$$

As a co-compact discrete subgroup of $G$, we can take for example

$$
\Gamma=6 \mathbb{Z} \times \mathbb{Z}^{4} .
$$

By the Nomizu's Theorem, $H_{D R}^{*}(M) \cong H^{*}(\mathfrak{g})$, where $H^{*}(\mathfrak{g})=H^{*}(\mathfrak{g}, \mathbb{R})$ denotes the Chevalley-Eilenberg cohomology of the Lie algebra $\mathfrak{g}$ with the coefficients in the trivial module $\mathbb{R}$.

By (3.2) we have that

$$
H^{1}(\mathfrak{g})=\left\langle\left[\alpha_{1}\right],\left[\alpha_{2}\right]\right\rangle
$$

Thus $b_{1}(M)=2$, so that the Betti numbers do not give any obstruction for $(M, \eta)$ to admit a Sasakian structure. Now, according to [5, Section 5], let us consider the Lefschetz relation

$$
\mathcal{R}_{L e f_{p}}=\left\{\left([\beta],\left[\eta \wedge(\mathrm{d} \eta)^{2-p} \wedge \beta\right]\right) \mid \beta \in \Omega^{p}(M), \mathrm{d} \beta=0, i_{\xi} \beta=0,(\mathrm{~d} \eta)^{3-p} \wedge \beta=0\right\} .
$$

As a consequence of [5, Theorem 4.5], if a 5-dimensional contact manifold $(M, \eta)$ admits any compatible Sasakian metric then $\mathcal{R}_{L e f_{p}}$ is the graph of an isomorphism for every $p \leq 2$. Actually, let us prove that in our case the above property does not hold for $p=1$. Let us consider the 1-form $\beta$ on $M$ induced by the left-invariant 1 -form on $G$ associated to $\alpha_{2}$. Clearly $\beta$ is closed, $i_{\xi} \beta=0$ and

$$
(\mathrm{d} \eta)^{2} \wedge \beta=0 \text {. }
$$


Moreover, using that $\left[\alpha_{2}\right]$ is a non-zero element of $H^{1}(\mathfrak{g})$, we deduce that $[\beta]$ is a non-zero element of $H_{D R}^{1}(M)$. We prove that

$$
[\eta \wedge \mathrm{d} \eta \wedge \beta]=0
$$

Indeed we have

$$
\mathrm{d}\left(\alpha_{3} \wedge \alpha_{4} \wedge \alpha_{5}\right)=-\alpha_{1} \wedge \alpha_{2} \wedge \alpha_{4} \wedge \alpha_{5}
$$

Therefore, $\eta \wedge \mathrm{d} \eta \wedge \beta$ is an exact 4-form on $M$. In fact, $\eta \wedge \mathrm{d} \eta \wedge \beta=-\mathrm{d} \gamma$, with $\gamma$ the 3form on $M$ induced by the left-invariant 3-form on $G$ associated to $\alpha_{3} \wedge \alpha_{4} \wedge \alpha_{5} \in \wedge^{3} \mathfrak{g}^{*}$. So, $\mathcal{R}_{\text {Lef }_{1}}$ is not the graph of an isomorphism and we conclude that $M$ cannot carry any (not necessarily left-invariant) Sasakian metric compatible with $\eta$.

Remark 3.3. We point out that though $(M, \eta)$ cannot admit a Sasakian metric, it admits a (even left-invariant) $K$-contact structure. Indeed, we can define an endomorphism $\phi: \mathfrak{g} \longrightarrow \mathfrak{g}$ by setting

$$
\phi X_{1}=-X_{4}, \quad \phi X_{2}=-X_{3}, \quad \phi X_{3}=X_{2}, \quad \phi X_{4}=X_{1}, \quad \phi X_{5}=0
$$

and we can define a positive definite bilinear form $g$ by declaring that $\left\{X_{1}, \ldots, X_{5}\right\}$ is $g$-orthonormal. Then $(\phi, \xi, \eta, g)$ induces a left-invariant contact metric structure on $G$ which descends to the quotient. Since $X_{5}$ belongs to the center of the Lie algebra $\mathfrak{g}$, we have immediately that the Reeb vector field $\xi$ is Killing and thus the structure is $K$-contact.

Example 3.4. Now, we shall present an example of a compact 7-dimensional $K$-contact manifold which does not admit any compatible Sasakian metric. As we shall see, one cannot deduce this fact by Theorem 2.1.

In a recent paper [11], Kutsak has classified all nilpotent 7-dimensional Lie algebras carrying a left-invariant contact structure. Let us consider the example (1457B) in that paper, namely the nilpotent Lie algebra $\mathfrak{g}$ with non-zero Lie brackets

$$
\begin{array}{ll}
{\left[X_{1}, X_{2}\right]=X_{3}, \quad\left[X_{1}, X_{3}\right]=X_{4}, \quad\left[X_{1}, X_{4}\right]=X_{7},} \\
{\left[X_{2}, X_{3}\right]=X_{7}, \quad\left[X_{5}, X_{6}\right]=X_{7} .}
\end{array}
$$

Let us denote by $\alpha_{i}$ the dual 1 -form of the vector $X_{i}$, for any $i \in\{1, \ldots, 7\}$. Then by (3.3) it follows that

$$
\begin{aligned}
& \mathrm{d} \alpha_{1}=0, \quad \mathrm{~d} \alpha_{2}=0, \quad \mathrm{~d} \alpha_{5}=0, \quad \mathrm{~d} \alpha_{6}=0, \quad \mathrm{~d} \alpha_{3}=-\alpha_{1} \wedge \alpha_{2}, \\
& \mathrm{~d} \alpha_{4}=-\alpha_{1} \wedge \alpha_{3} \quad \mathrm{~d} \alpha_{7}=-\alpha_{1} \wedge \alpha_{4}-\alpha_{2} \wedge \alpha_{3}-\alpha_{5} \wedge \alpha_{6} .
\end{aligned}
$$

Let $G$ be the simply connected Lie group $G$ with Lie algebra $\mathfrak{g}$ and consider the left-invariant 1-form on $G$ associated to $\alpha_{7}$. Then, such a 1 -form defines a contact structure on $G$. The corresponding Reeb vector field $\xi$ is the left-invariant vector field on $G$ induced by $X_{7}$.

Now, let us consider a co-compact discrete subgroup $\Gamma$ of $G$. Then the quotient $M=G / \Gamma$ is a compact nilmanifold. 
Remark 3.5. The Lie group $G$ is isomorphic to $\mathbb{R}^{7}$ endowed with the multiplication defined by

$$
\begin{aligned}
&\left(x^{1}, x^{2}, x^{3}, x^{4}, x^{5}, x^{6}, x^{7}\right) \cdot\left(y^{1}, y^{2}, y^{3}, y^{4}, y^{5}, y^{6}, y^{7}\right)=\left(x^{1}+y^{1}, x^{2}+y^{2}, x^{3}+y^{3}+x^{1} y^{2},\right. \\
& x^{4}+y^{4}+x^{1} y^{3}+\frac{\left(x^{1}\right)^{2}}{2} y^{2}, x^{5}+y^{5}, x^{6}+y^{6}, x^{7}+y^{7}+x^{1} y^{4} \\
&\left.+\left(\frac{\left(x^{1}\right)^{2}}{2}+x^{2}\right) y^{3}+\frac{x^{1}}{2}\left(y^{2}\right)^{2}+\left(\frac{\left(x^{1}\right)^{3}}{6}+x^{1} x^{2}\right) y^{2}+x^{5} y^{6}\right),
\end{aligned}
$$

for $\left(x^{1}, x^{2}, x^{3}, x^{4}, x^{5}, x^{6}, x^{7}\right),\left(y^{1}, y^{2}, y^{3}, y^{4}, y^{5}, y^{6}, y^{7}\right) \in \mathbb{R}^{7}$. A basis of the space of the left-invariant 1 -forms is given by

$$
\begin{aligned}
& \alpha_{1}=\mathrm{d} x^{1}, \quad \alpha_{2}=\mathrm{d} x^{2}, \quad \alpha_{3}=\mathrm{d} x^{3}-x^{1} \mathrm{~d} x^{2}, \quad \alpha_{4}=\mathrm{d} x^{4}-x^{1} \mathrm{~d} x^{3}+\frac{\left(x^{1}\right)^{2}}{2} \mathrm{~d} x^{2}, \\
& \alpha_{5}=\mathrm{d} x^{5}, \alpha_{6}=\mathrm{d} x^{6}, \alpha_{7}=\mathrm{d} x^{7}-x^{1} \mathrm{~d} x^{4}+\frac{\left(x^{1}\right)^{2}}{2} \mathrm{~d} x^{3}-\frac{\left(x^{1}\right)^{3}}{6} \mathrm{~d} x^{2}-x^{2} \mathrm{~d} x^{3}-x^{5} \mathrm{~d} x^{6} .
\end{aligned}
$$

A co-compact discrete subgroup of $G$ is

$$
\Gamma=6 \mathbb{Z} \times \mathbb{Z}^{6} .
$$

By the Nomizu's Theorem $H_{D R}^{*}(M) \cong H^{*}(\mathfrak{g})$. By (3.4) we have that $H^{1}(\mathfrak{g})=$ $\left\langle\left[\alpha_{1}\right],\left[\alpha_{2}\right],\left[\alpha_{5}\right],\left[\alpha_{6}\right]\right\rangle$. Thus $b_{1}(M)=4$. Furthermore, by using the library [14] of the computer algebra system Singular [7, one can compute the dimension of $H^{3}(\mathfrak{g})$. Namely, the cohomology group $H^{3}(\mathfrak{g}, \mathbb{R})$ is isomorphic to the third Ext-group $\operatorname{Ext}_{\mathfrak{U}(\mathfrak{g})}^{3}(\mathbb{R}, \mathbb{R})$, where $\mathfrak{U}(\mathfrak{g})$ denotes the universal enveloping algebra of $\mathfrak{g}$. Note that the Lie algebra $\mathfrak{g}$ is a positively graded Lie algebra with the grading

$$
\begin{array}{lll}
\operatorname{deg}\left(X_{1}\right)=\operatorname{deg}\left(X_{5}\right)=1, & \operatorname{deg}\left(X_{2}\right)=2, & \operatorname{deg}\left(X_{3}\right)=3, \\
\operatorname{deg}\left(X_{4}\right)=\operatorname{deg}\left(X_{6}\right)=4, & \operatorname{deg}\left(X_{7}\right)=5 . &
\end{array}
$$

Therefore, we can consider $\mathfrak{U}(\mathfrak{g})$ as a connected graded associative algebra. Thus the dimension of $\operatorname{Ext}_{\mathfrak{U}(\mathfrak{g})}^{k}(\mathbb{R}, \mathbb{R})$ coincides with the rank of the (necessarily free) $k$-th module of the minimal projective resolution of $\mathbb{R}$ over $\mathfrak{U}(\mathfrak{g})$. One can use the procedure mres of Singular to construct such a resolution. As a result, we get that $b_{3}(M)=8$. Therefore, the dimension of $H^{3}(\mathfrak{g})$ does not give any obstruction to the existence of a Sasakian structure on $(M, \eta)$, where $\eta$ is the contact 1-form on $M$ induced by the left-invariant 1-form on $G$ associated to $\alpha_{7}$.

Now, according to [5, Section 5], let us consider the Lefschetz relation

$$
\mathcal{R}_{L e f_{p}}=\left\{\left([\beta],\left[\eta \wedge(\mathrm{d} \eta)^{3-p} \wedge \beta\right]\right) \mid \beta \in \Omega^{p}(M), \mathrm{d} \beta=0, i_{\xi} \beta=0,(\mathrm{~d} \eta)^{4-p} \wedge \beta=0\right\} .
$$

As recalled in Section 2] as a consequence of [5, Theorem 4.5], if a 7-dimensional contact manifold $(M, \eta)$ admits any compatible Sasakian metric then $\mathcal{R}_{\text {Lef }_{p}}$ is the graph of an isomorphism for every $p \leq 3$. Actually, let us prove that in our case the above property does not hold for $p=1$. Let us consider the 1-form $\beta$ on $M$ induced by the left-invariant 1 -form on $G$ associated to $\alpha_{1} \in \mathfrak{g}^{*}$. First of all let us check that $\mathcal{R}_{L e f_{1}}$ is defined for $\beta$. Indeed, $\beta$ is closed, $i_{\xi} \beta=0$ and

$$
(\mathrm{d} \eta)^{3} \wedge \beta=0 .
$$

Clearly, $[\beta]$ defines a non-zero element of $H_{D R}^{1}(M)$. Finally

$$
\left[\eta \wedge(\mathrm{d} \eta)^{2} \wedge \beta\right]=0 .
$$


Indeed we have

$$
\begin{aligned}
\alpha_{7} \wedge\left(\mathrm{d} \alpha_{7}\right)^{2} \wedge \alpha_{1} & =-2 \alpha_{1} \wedge \alpha_{2} \wedge \alpha_{3} \wedge \alpha_{5} \wedge \alpha_{6} \wedge \alpha_{7} \\
& =2 \mathrm{~d}\left(\alpha_{2} \wedge \alpha_{4} \wedge \alpha_{5} \wedge \alpha_{6} \wedge \alpha_{7}\right) .
\end{aligned}
$$

Therefore $\mathcal{R}_{L e f_{1}}$ is not the graph of an isomorphism and we conclude that $M$ cannot carry any (not necessarily left-invariant) Sasakian metric compatible with $\eta$.

Remark 3.6. We point out that though $(M, \eta)$ cannot admit a Sasakian metric, it admits a (even left-invariant) $K$-contact structure. Indeed, we can define an endomorphism $\phi: \mathfrak{g} \longrightarrow \mathfrak{g}$ by setting

$$
\begin{aligned}
& \phi X_{1}=X_{4}, \quad \phi X_{2}=X_{3}, \quad \phi X_{3}=-X_{2}, \quad \phi X_{4}=-X_{1}, \\
& \phi X_{5}=X_{6}, \quad \phi X_{6}=-X_{5}, \quad \phi X_{7}=0 .
\end{aligned}
$$

and we can define a positive definite bilinear form $g$ by declaring that $\left\{X_{1}, \ldots, X_{7}\right\}$ is $g$-orthonormal. Then $\left(\phi, X_{7}, \alpha_{7}, g\right)$ induces a left-invariant contact metric structure on $G$ which descends to the quotient. Since $X_{7}$ belongs to the center of the Lie algebra $\mathfrak{g}$, we have immediately that the Reeb vector field is Killing and thus the structure is $K$-contact.

Remark 3.7. In [1] it was proved that every nilpotent Lie algebra admitting a Sasakian structure is necessarily isomorphic to the Heisenberg Lie algebra. Thus one could think that the non-Sasakian property for our examples can be deduced from this result. Actually, our results are stronger, because we prove that the nilmanifolds in Examples 3.1 and 3.4 cannot admit any compatible Sasakian structure, not necessarily left-invariant.

\section{Acknowledgments}

Research partially supported by CMUC, funded by the European program COMPETE/FEDER, by FCT (Portugal) grants PEst-C/MAT/UI0324/2011 (A.D.N. and I.Y.), by MICINN (Spain) grants MTM2011-15725-E, MTM2012-34478 (A.D.N. and J.C.M.), the project of the Canary Government ProdID20100210 (J.C.M.), and by Prin 2010/11 - Varietà reali e complesse: geometria, topologia e analisi armonica Italy (B.C.M.).

\section{REFERENCES}

[1] A. Andrada, A. Fino, L. Vezzoni, A class of Sasakian 5-manifolds, Transformation Groups 14 (2009), 493-512.

[2] C. Benson, C. S. Gordon, Kähler and symplectic structures on nilmanifolds, Topology 27 (1988), 513-518.

[3] D. E. Blair, Riemannian geometry of contact and symplectic manifolds, Second Edition. Progress in Mathematics 203, Birkhäuser, Boston, 2010.

[4] C. P. Boyer and K. Galicki, Sasakian geometry, Oxford University Press, 2008.

[5] B. Cappelletti-Montano, A. De Nicola, I. Yudin, Hard Lefschetz Theorem for Sasakian manifolds, arXiv:1306.2896 1.

[6] D. Chinea, M. de León, and J. C. Marrero, Topology of cosymplectic manifolds, J. Math. Pures Appl. (9) 72 (1993), no. 6, 567-591.

[7] W. Decker, G.-M. Greuel, G. Pfister, H. Schönemann, Singular 3-1-5 - A computer algebra system for polynomial computations, http://www.singular.uni-kl.de, 2012.

[8] T. Fujitani, Complex-valued differential forms on normal contact Riemannian manifolds, Tôhoku Math. J. 18 (1966), 349-361.

[9] B. Hajduk, A. Tralle, On simply connected K-contact non-Sasakian manifolds, arXiv:1305.2591v1. 
[10] I. Kolár, P. Michor, J. Slovák, Natural operations in differential geometry, Springer-Verlag, Berlin, 1993.

[11] S. Kutsak, Invariant contact structures on 7-dimensional nilmanifolds, Geom. Dedicata 172 (2014), 351-361.

[12] E. Lerman, How fat is a fat bundle?, Lett. Math. Phys. 15 (1988), 335-339.

[13] E. Lerman, Contact fiber bundles, J. Geom. Phys. 49 (2004), 52-66.

[14] V. Levandovskyy, F. L. Lobillo, C. Rabelo, O. Motsak, nctools.lib. A Singular 3-1-5 library of general tools for noncommutative algebras, 2013.

[15] Y. Lin, Lefschetz contact manifolds and odd dimensional symplectic geometry, arXiv:1311.1431v2.

[16] W. P. Thurston, Some simple examples of symplectic manifolds, Proc. Amer. Math. Soc., 55 (1976), 467-468.

[17] A. Tralle, G. Oprea, Symplectic manifolds with no Kähler structure, Lecture Notes in Mathematics 1661, Springer-Verlag, Berlin, 1997.

Dipartimento di Matematica e Informatica, Università degli Studi di Cagliari, Via OsPedale 72, 09124 Cagliari, Italy

E-mail address: b.cappellettimontano@gmail.com

CMUC, Department of Mathematics, University of Coimbra, 3001-501 Coimbra, PortuGAL

E-mail address: antondenicola@gmail.com

Unidad Asociada UlL-CSiC "Geometría Diferencial y Mecánica Geométrica” Departamento de Matemáticas, Estadística e Investigación Operativa, Facultad de Ciencias, Universidad de La Laguna, La Laguna, Tenerife, Spain

E-mail address: jcmarrer@ull.es

CmuC, Department of Mathematics, University of Coimbra, 3001-501 Coimbra, PortuGAL

E-mail address: yudin@mat.uc.pt 